\title{
Cooperative Multiple Access under Energy Harvesting Constraints
}

\author{
Nuğman $\mathrm{Su}^{1}$, Onur Kaya ${ }^{2}$, Sennur Ulukus ${ }^{3}$ and Mutlu Koca ${ }^{1}$ \\ ${ }^{1}$ Boğaziçi University, ${ }^{2}$ Işık University, ${ }^{3}$ University of Maryland
}

\begin{abstract}
We consider a cooperative multiple access channel (MAC) with two energy harvesting transmitters. The transmitters perform delay constrained transmission, i.e., every information block is encoded, transmitted and decoded between two consecutive energy harvests. We aim to maximize the achievable departure region over a finite transmission duration. We formulate the departure region maximization as a convex optimization problem. We propose an iterative algorithm which uses a directional waterfilling strategy to calculate the optimal power components. The departure region obtained by cooperation is shown to be significantly larger than that of a MAC without cooperation under the same energy arrival patterns. As a special case, we also analyze an energy harvesting relay channel with full duplex cooperation.
\end{abstract}

\section{INTRODUCTION}

We consider an energy harvesting communication system where the transmitters harvest energy from nature to sustain power needed for their data transmission. In such systems, energy is harvested (arrives at the transmitter) during the course of communication, intermittently, and in varying amounts. Recent work has considered information theoretic capacity of such systems, [1], [2], as well as optimum data scheduling in such systems by assuming a concave rate-power relationship [3]-[6]. The scheduling literature has been extended to multiuser scenarios such as multiple access channels [7], [8], broadcast channels [9]-[11], interference channels [12], twohop channels [13]-[15]. In this paper, we extend this line of work to the cooperative multiple access channel (MAC) [16].

In a cooperative MAC, users over-hear each other's transmission, form common messages out of these over-heard signals by decoding, and beamform data to the receiver, achieving higher throughputs for the system. Such cooperation schemes exploit the difference in channel strengths of the two users to the benefit of the system, by routing data through stronger channels among the users. In fading channels, the achievable rates can be further improved by instantaneous channel-state based power control that exploits better channel states of the system over time [17]. Such approaches result in multi-user diversity and time-diversity by exploiting variations of the channel gains from user to user and over time.

For the energy harvesting MAC, even for a non-fading system, there is variation in the transmission capabilities of the users due to the time-varying nature of their available energies, thereby creating a new form of diversity called energy diversity. In this paper, we consider a slotted energy arrival

This work was supported by TUBITAK grant 113E556.

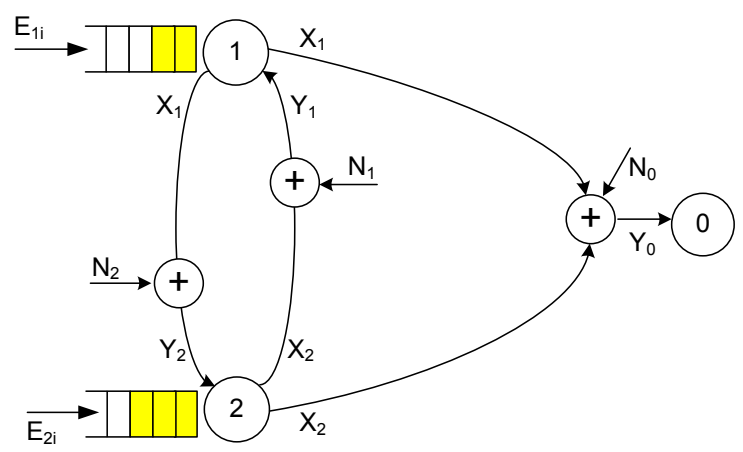

Fig. 1. Energy harvesting cooperative MAC model.

and cooperative transmission model for the energy harvesting MAC, determine achievable rates as functions of the powers of the users, and optimize these powers subject to energy harvesting constraints in order to maximize the departure region of the system. Therefore, we determine transmit power sequences for the users that take advantage of not only the stronger channels but also the stronger battery energy states due to energy harvesting, through physical layer cooperation. Our work generalizes the power control framework for the energy harvesting MAC in [7] to the case of user cooperation, and two-hop energy harvesting systems with a dedicated relay in [13]-[15] to the case of a two-sided cooperation setting. Our simulation results indicate significant gains over no cooperation and one-sided cooperation.

\section{SySTEM MODEL}

We consider a cooperative MAC model with energy harvesting transmitters as shown in Fig. 1. We assume a slotted energy arrival model, where a non-zero energy packet, $E_{k i}$, arrives at user $k$ at the beginning of each slot $i$; with $k=1,2$ and $i=1, \ldots, N$. The incoming energy packets at each user are used only for transmission purposes and can be stored in the energy buffer of that user for later use. The slots are assumed to be of fixed length $T$. We assume unit-length slots without loss of generality, so that we can use power and energy interchangeably.

The signals observed in the $i$ th slot at the receiver (denoted as node 0 ), user 1 and user 2, respectively, are given by

$$
\begin{aligned}
\mathbf{Y}_{0 i} & =\mathbf{X}_{1 i}+\mathbf{X}_{2 i}+\mathbf{N}_{0 i} \\
\mathbf{Y}_{1 i} & =\mathbf{X}_{2 i}+\mathbf{N}_{1 i} \\
\mathbf{Y}_{2 i} & =\mathbf{X}_{1 i}+\mathbf{N}_{2 i}
\end{aligned}
$$


where $\mathbf{X}_{k i}$ is the transmitted codeword by user $k$ and $\mathbf{N}_{0 i}, \mathbf{N}_{1 i}$ and $\mathbf{N}_{2 i}$ denote the additive white Gaussian noise (AWGN) terms at the respective nodes. The signal model is normalized such that the noise components $\mathbf{N}_{1 i}$ and $\mathbf{N}_{2 i}$ are i.i.d. with unit-variance. The noise components $\mathbf{N}_{0 i}$ at the receiver are i.i.d. with variance $\sigma^{2}$. We assume that $\sigma^{2}>1$, meaning that the inter-user links are stronger than the direct links.

During each slot, each user accesses the channel $n B$ times, where $n, B \rightarrow \infty$. In each slot $i$ and sub-block $b$, $b=1, \ldots, B$; each user $k$ sends the message $w_{k i}(b)$, using the length- $n$ codeword $\mathbf{x}_{k j i}(b) \triangleq \mathbf{x}_{k j i}\left(w_{k i}(b)\right)$ to its cooperating partner $j, j=1,2, j \neq k$. Meanwhile, users $k$ and $j$ decode the messages $w_{j i}(b-1)$ and $w_{k i}(b-1)$, respectively, exchanged in the previous sub-block and form the length $n$ common codeword $\mathbf{u}_{i}(b) \triangleq \mathbf{u}_{i}\left(w_{k i}(b-1), w_{j i}(b-1)\right)$. While it is also possible for the users to send private messages to the receiver, such approach is known to be suboptimal when $\sigma^{2}>1$ [17], and is therefore not adopted here. Codewords $\mathbf{x}_{k j i}(b)$ and $\mathbf{u}_{i}(b)$ are superposed and transmitted over each sub-block $b$. Letting $\mathbf{X}_{k j i}=\left[\mathbf{x}_{k j i}(1), \ldots, \mathbf{x}_{k j i}(B)\right]$ and $\mathbf{U}_{i}=\left[\mathbf{u}_{i}(1), \ldots, \mathbf{u}_{i}(B)\right]$, the transmitted codewords by the users over each slot $i$ can then be written as,

$$
\begin{aligned}
& \mathbf{X}_{1 i}=\sqrt{p_{12 i}} \mathbf{X}_{12 i}+\sqrt{p_{U_{1} i}} \mathbf{U}_{i}, \quad i=1, \ldots, N, \\
& \mathbf{X}_{2 i}=\sqrt{p_{21 i}} \mathbf{X}_{21 i}+\sqrt{p_{U_{2} i}} \mathbf{U}_{i}, \quad i=1, \ldots, N,
\end{aligned}
$$

where $p_{k j i}$ and $p_{U_{k i}}$ denote the powers associated with each codeword in slot $i$. These powers need to be kept constant in each slot, as will be proved later. Decoding at the receiver takes place at the end of each slot, after receiving the block codewords of length $n B$, by using backwards decoding. This strategy results in common data created in slot $i$, to be transmitted in slot $i$, hence the delay constrained model. The transmit powers $p_{k j i}$ and $p_{U_{k i}}, \forall i$, must satisfy

$$
\begin{aligned}
& \sum_{i=1}^{\ell} p_{12 i}+p_{U_{1} i} \triangleq \sum_{i=1}^{\ell} p_{1 i} \leq \sum_{i=1}^{\ell} E_{1 i}, \quad \ell=1, \ldots, N \\
& \sum_{i=1}^{\ell} p_{21 i}+p_{U_{2} i} \triangleq \sum_{i=1}^{\ell} p_{2 i} \leq \sum_{i=1}^{\ell} E_{2 i}, \quad \ell=1, \ldots, N .
\end{aligned}
$$

Here, (6)-(7) follow from the energy causality constraints [3], which state that the cumulative energy spent by any slot should not exceed the cumulative energy harvested up to and including that slot.

In the next section, we formulate the departure region maximization problem [7] for the energy harvesting cooperative MAC and solve it in terms of the transmit powers for any given energy arrival pattern.

\section{Achievable Departure Region and Optimum TRANSMIT / COOPERATION SCHEDULING}

We begin by characterizing the departure region achievable by the encoding/decoding policy proposed in Section II. Let $\mathbf{p} \triangleq\left\{\mathbf{p}_{12}, \mathbf{p}_{21}, \mathbf{p}_{U_{1}}, \mathbf{p}_{U_{2}}\right\}$ denote the vector of transmit powers, each entry being a row vector containing the subpowers for all slots $i=1, \ldots, N$. For the model described by
(1)-(3), and a feasible power vector satisfying (6)-(7), the rate pair $\left(R_{1 i}, R_{2 i}\right)$ in slot $i$ is achievable if it satisfies

$$
\begin{aligned}
R_{1 i} & <\frac{1}{2} \log \left(1+p_{12 i}\right), \\
R_{2 i} & <\frac{1}{2} \log \left(1+p_{21 i}\right), \\
R_{1 i}+R_{2 i} & <\frac{1}{2} \log \left(\frac{S_{i}}{\sigma^{2}}\right),
\end{aligned}
$$

where $S_{i} \triangleq \sigma^{2}+p_{1 i}+p_{2 i}+2 \sqrt{p_{U_{1} i} p_{U_{2}} i}$. The total number of bits transmitted by each user $k$ is given by $B_{k}=\sum_{i} R_{k i}$. The achievable departure region is then defined as the set of $\left(B_{1}, B_{2}\right)$ pairs that can simultaneously be supported under the rate constraints (8)-(10) and the energy causality constraints (6)-(7). The departure region is convex due to the possibility of time-sharing. Therefore, for any given energy pattern, points on the departure region can be obtained by maximizing

$$
B_{\boldsymbol{\mu}} \triangleq \mu_{1} B_{1}+\mu_{2} B_{2}=\sum_{i}\left(\mu_{1} R_{1 i}+\mu_{2} R_{2 i}\right) \triangleq \sum_{i} R_{\mu i}
$$

for given priorities $0 \leq \mu_{1} \leq 1$ and $0 \leq \mu_{2} \leq 1$. By varying $\left(\mu_{1}, \mu_{2}\right)$, and taking the convex hull of resulting boundary points, the departure region can be formed. Incorporating the rate constraints (8)-(10) into the cost function (11), the objective function $B_{\mu}$ can be written in closed-form as,

$$
\begin{aligned}
B_{\boldsymbol{\mu}}= & \sum_{i=1}^{N} \frac{\mu_{1}-\mu_{2}}{2} \min \left\{\log \left(1+p_{12 i}\right), \log \left(\frac{S_{i}}{\sigma^{2}}\right)\right\} \\
& +\frac{\mu_{2}}{2} \min \left\{\log \left(\frac{S_{i}}{\sigma^{2}}\right), \log \left(1+p_{12 i}\right)+\log \left(1+p_{21 i}\right)\right\},
\end{aligned}
$$

where $\mu_{1}>\mu_{2}$ is assumed without loss of generality. Before stating and solving the departure region maximization problem, we will prove some key properties of the objective function, and the power allocation policies maximizing it.

Lemma 1 The weighted departure function $B_{\boldsymbol{\mu}}$ is concave in the power vector $\mathbf{p}, \forall\left(\mu_{1}, \mu_{2}\right)$.

Proof: Since a linear combination of concave functions is concave, it suffices to show each term in (12) is concave in $\mathbf{p}$. The functions $\log \left(1+p_{12 i}\right)$ and $\log \left(1+p_{21 i}\right)$ are obviously concave in $\mathbf{p}$. It is also easy to show that $2 \sqrt{p_{U_{1} i} p_{U_{2} i}}$ is concave and increasing in $\mathbf{p}$, making $\frac{S_{i}}{\sigma^{2}}$ concave and increasing in $\mathbf{p}$. The term $\log \left(\frac{S_{i}}{\sigma^{2}}\right)$, being a composition of the concave logarithm with concave and increasing $\frac{S_{i}}{\sigma^{2}}$, is therefore concave. The result then follows by noting that the minimum of two concave functions is concave.

The following corollary justifies the use of constant transmit powers in each slot, for each sub-codeword.

Corollary 1 The transmit powers $p_{12 i}, p_{21 i}, p_{U_{1} i}$ and $p_{U_{2} i}$ for each sub-codeword in a given slot $i$ must be kept constant throughout the slot.

The proof of Corollary 1 follows from the concavity of (12), using a similar argument to [3, Lemma 2]. 
We are now ready to solve the departure region maximization problem, which can be formally stated as

$$
\begin{aligned}
\mathbf{P 1}: \max _{\mathbf{p}} & \sum_{i=1}^{N} R_{\mu i} \\
\text { s.t. } & \sum_{i=1}^{\ell} p_{12 i}+p_{U_{1} i} \leq \sum_{i=1}^{\ell} E_{1 i}, \quad \ell=1, \ldots, N, \\
& \sum_{i=1}^{\ell} p_{21 i}+p_{U_{2} i} \leq \sum_{i=1}^{\ell} E_{2 i}, \quad \ell=1, \ldots, N, \\
& R_{\mu i} \leq \frac{\mu_{1}}{2} \log \left(1+p_{12 i}\right)+\frac{\mu_{2}}{2} \log \left(1+p_{21 i}\right), \\
& R_{\mu i} \leq \frac{\mu_{1}-\mu_{2}}{2} \log \left(1+p_{12 i}\right)+\frac{\mu_{2}}{2} \log \left(\frac{S_{i}}{\sigma^{2}}\right), \\
& p_{12 i}, p_{U_{1} i}, p_{21 i}, p_{U_{2} i} \geq 0, \quad i=1, \ldots, N .
\end{aligned}
$$

The problem $\mathbf{P 1}$ consists of $2 N$ convex rate constraints and $2 N$ affine energy causality constraints, and $4 N$ non-negativity constraints. The objective function is affine, hence $\mathbf{P 1}$ is a convex optimization problem. Assigning non-negative Lagrange multipliers $\lambda_{1 i}, \lambda_{2 i}, \gamma_{1 i}$ and $\gamma_{2 i}$ to the constraints (14)(17), respectively, and further invoking the complementary slackness conditions for the non-negativity constraints (18), the KKT conditions, which are necessary and sufficient for optimality can be obtained for each $i$ as follows:

$$
\begin{aligned}
& \gamma_{1 i}+\gamma_{2 i}=1, \\
& \sum_{\ell=i}^{N} \lambda_{1 \ell} \geq \frac{\gamma_{1 i} \mu_{1}}{2\left(1+p_{12 i}\right)}+\frac{\gamma_{2 i}\left(\mu_{1}-\mu_{2}\right)}{2\left(1+p_{12 i}\right)}+\frac{\gamma_{2 i} \mu_{2}}{2 S_{i}}, \\
& \sum_{\ell=i}^{N} \lambda_{1 \ell} \geq \frac{\gamma_{2 i} \mu_{2}\left(\sqrt{p_{U_{1} i}}+\sqrt{p_{U_{2}}}\right)}{2 S_{i} \sqrt{p_{U_{1} i}}}, \\
& \sum_{\ell=i}^{N} \lambda_{2 \ell} \geq \frac{\gamma_{1 i} \mu_{2}}{2\left(1+p_{21 i}\right)}+\frac{\gamma_{2 i} \mu_{2}}{2 S_{i}}, \\
& \sum_{\ell=i}^{N} \lambda_{2 \ell} \geq \frac{\gamma_{2 i} \mu_{2}\left(\sqrt{p_{U_{1} i}}+\sqrt{p_{U_{2}} i}\right)}{2 S_{i \sqrt{p_{U_{2}}}}} .
\end{aligned}
$$

The inequalities in (20)-(23) are satisfied with equality when the corresponding power variable, with respect to which the derivative is taken is positive, and are loose otherwise. However, the optimality conditions (19)-(23) are clearly coupled, and each power component of each user depends on the strategy used by the other user. Moreover, whether a user should use its power to cooperate in a slot or save it for cooperation in later slots is not immediately evident from (19)-(23). In what follows, we first prove a key lemma which enables us to obtain a relation among optimal power components, which holds irrespective of the values of the Lagrange multipliers. Using this relation, we will propose an iterative solution based on the KKT conditions, which iterates over the component power calculation, and energy flow to future slots.

Lemma 2 The optimum power allocation policy which maximizes the weighted departure function can be found by focusing only on policies which satisfy

$$
\left(1+p_{12 i}\right)\left(1+p_{21 i}\right)=\left(\frac{S_{i}}{\sigma^{2}}\right) .
$$

Moreover, for the special case of throughput maximization with $\mu_{1}=\mu_{2}=1$, the optimum policy must satisfy (24) and the cooperative powers $p_{U_{1} i}, p_{U_{2} i}$ in each slot must be non-zero.

Proof: We first prove the second part. With $\mu_{1}=\mu_{2}=1$, the first term in (12) disappears. The second term is maximized when the arguments of the minimum operation are equal. To see this, assume we know the optimal total power levels in a given slot are such that $\log \left(1+p_{12 i}\right)+\log (1+$ $\left.p_{21 i}\right)>\log \left(\frac{S_{i}}{\sigma^{2}}\right)$. While keeping the total energy consumed by each user in slot $i$ constant, we can transfer some of the powers $p_{12 i}$ and $p_{21 i}$ to $p_{U_{1} i}$ and $p_{U_{2} i}$, respectively, until $\log \left(1+p_{12 i}\right)+\log \left(1+p_{21 i}\right)=\log \left(\frac{S_{i}}{\sigma^{2}}\right)$. This clearly increases the minimum of the two terms, which contradicts the optimality of the original powers. A similar contradiction argument can be used for the opposite ordering. Since the two functions are guaranteed to intersect, the maximum is always attained when the two arguments are equal. Moreover, since $\sigma^{2}>1$, setting either one of $p_{U_{1} i}$ or $p_{U_{2} i}$ to zero results in $\log \left(1+p_{12 i}\right)+\log \left(1+p_{21 i}\right)>\log \left(\frac{S_{i}}{\sigma^{2}}\right)$; meaning that the optimal transmission policy is to transmit cooperative signals regardless of the energy arrival pattern or priorities.

For arbitrary $\mu_{1}$ and $\mu_{2}$, the result of the first minimum operation in (12) must necessarily be $\log \left(1+p_{12 i}\right)$, or else both of the terms in (12) could be increased, or at least held constant, by increasing $p_{U_{1} i}$ in lieu of $p_{12 i}$. The ordering $\log \left(\frac{S_{i}}{\sigma^{2}}\right)>\log \left(1+p_{12 i}\right)+\log \left(1+p_{21 i}\right)$ is not possible, or else $\log \left(1+p_{12 i}\right)+\log \left(1+p_{21 i}\right)$ can be further increased by decreasing $p_{U_{1} i}$. Then, at the optimal point we must have $\log \left(1+p_{12 i}\right) \leq \log \left(\frac{S_{i}}{\sigma^{2}}\right) \leq \log \left(1+p_{12 i}\right)+\log \left(1+p_{21 i}\right)$. In order to maximize (12), either the first or second inequality must be tight. If the second inequality is tight, the proof is complete. If the first equality is tight, the same overall weighted departure can be achieved by setting $p_{21 i}=0$, which makes the second inequality also tight, which completes the proof.

The optimum departure region can now be attained by iterating the following two stage procedure:

Stage 1: Power distribution within slots: Assume we have a fixed feasible initial power allocation $\left[p_{1 \ell}, p_{2 \ell}\right]$ for slot $\ell$, determined by the amount of energy allocated to that slot. Assuming that all sub-power components are positive, the conditions (20)-(23) are satisfied with equality. Using (19)(23), we obtain

$\frac{\mu_{1}}{\mu_{2}}=\frac{\sqrt{p_{U_{1} \ell} p_{U_{2} \ell}}\left(\sigma^{2}+p_{1 \ell}+p_{2 \ell}\right)+p_{U_{1} \ell} p_{U_{2} \ell}+p_{U_{2} \ell}\left(1+p_{1 \ell}\right)}{\sqrt{p_{U_{1} \ell} p_{U_{2} \ell}}\left(\sigma^{2}+p_{1 \ell}+p_{2 \ell}\right)+p_{U_{1} \ell} p_{U_{2} \ell}+p_{U_{1} \ell}\left(1+p_{2 \ell}\right)}$.

With fixed $\left[p_{1 \ell}, p_{2 \ell}\right]$, this yields a quadratic form for $\sqrt{p_{U_{1} \ell}}$, in terms of the only remaining unknown, $p_{U_{2} \ell}$, i.e.,

$$
A p_{U_{1} \ell}+B \sqrt{p_{U_{1} \ell}}+C=0,
$$


with

$$
\begin{aligned}
& A=\mu_{1}\left(p_{2 \ell}+p_{U_{2} \ell}+1\right)-\mu_{2} p_{U_{2} \ell}, \\
& B=\left(\mu_{1}-\mu_{2}\right) \sqrt{p_{U_{2} \ell}}\left(\sigma^{2}+p_{1 \ell}+p_{2 \ell}\right), \\
& C=-\mu_{2} p_{U_{2} \ell}\left(1+p_{1 \ell}\right) .
\end{aligned}
$$

Now, using the condition (24) posed in Lemma 2, $p_{U_{1} \ell}$ and $p_{U_{2} \ell}$ can be found by jointly solving the equations given in (24) and (25) simultaneously. This gives the optimum distribution of sub-powers for a given pair of $p_{1 \ell}$ and $p_{2 \ell}$. In case there is no joint solution for (24) and (25) for given $p_{1 \ell}$ and $p_{2 \ell}$, this means at least one of the sub-powers is equal to zero and the corresponding KKT condition is satisfied with strict inequality. Using the arguments in the proof of Lemma $2, p_{21}$ (or $p_{12}$, if $\mu_{2} \geq \mu_{1}$ ) is set to 0 , and the non-zero subpowers are found by (24).

Stage 2: Energy flow among slots: Once the subpowers are determined, the Lagrange multipliers $\gamma_{1 \ell}$ and $\gamma_{2 \ell}$ are found by equating the right hand sides of (20) and (21) (or (22) and (23)) and using (19). Then, the expressions $\left(\sum_{i=\ell}^{N} \lambda_{k i}\right)^{-1}$ can be obtained $\forall \ell$. Let us define $v_{k \ell} \triangleq\left(\sum_{i=\ell}^{N} \lambda_{k i}\right)^{-1}$, which can be interpreted as the generalized water level in slot $\ell$, to be directionally filled to future slots, if necessary. Since $\lambda_{k i} \geq 0$ by definition, $v_{k \ell}$ must be increasing with $\ell$. At the end of stage 1 , the water level for slot $\ell$ is found by $v_{k \ell}=\left(\sum_{i=\ell}^{N} \lambda_{k i}\right)^{-1}$. If $v_{k \ell}>v_{k(\ell+1)}$, there needs to be an energy flow from slot $\ell$ to $\ell+1$. The amount of energy flow required is determined by re-distributing the powers using stage 1 , and repeating the iteration among stages 1 and 2 until the KKT conditions are simultaneously satisfied.

With the two stages defined above, the algorithm that results in the optimum power allocation for the cooperative MAC is summarized in Algorithm 1. The resulting departure region, along with the corresponding power distributions, will be demonstrated in the simulation results section.

\section{ONE Sided CoOperation: The Full-DupleX GAUSSIAN RELAY}

In this section, we consider the special case where user 2 is a dedicated relay for user 1 (the source). The channel model is re-defined here for direct comparison with previous work [14]: the source relay and relay destination link gains are unity whereas the link between the destination and the source is weaker $\left(h_{10}<1\right)$. The noise variances are also set to unity. The signal model in this modified framework is given by

$$
\begin{aligned}
& \mathbf{Y}_{0 i}=\sqrt{h_{10}} \mathbf{X}_{1 i}+\mathbf{X}_{2 i}+\mathbf{N}_{0 i} \\
& \mathbf{Y}_{2 i}=\mathbf{X}_{1 i}+\mathbf{N}_{2 i} .
\end{aligned}
$$

The transmitted codewords by both users are

$$
\begin{array}{ll}
\mathbf{X}_{1 i}=\sqrt{p_{12 i}} \mathbf{X}_{12 i}+\sqrt{p_{U_{1} i}} \mathbf{U}_{i}, & i=1, \ldots, N, \\
\mathbf{X}_{2 i}=\sqrt{p_{U_{2} i}} \mathbf{U}_{i}, & i=1, \ldots, N,
\end{array}
$$

where the only purpose of the relay is to form $\mathbf{u}_{i}(b)$ in every sub-block and transmit it to the destination. The optimization problem $\mathbf{P 2}$ for maximizing the rate of user 1, can be formu-

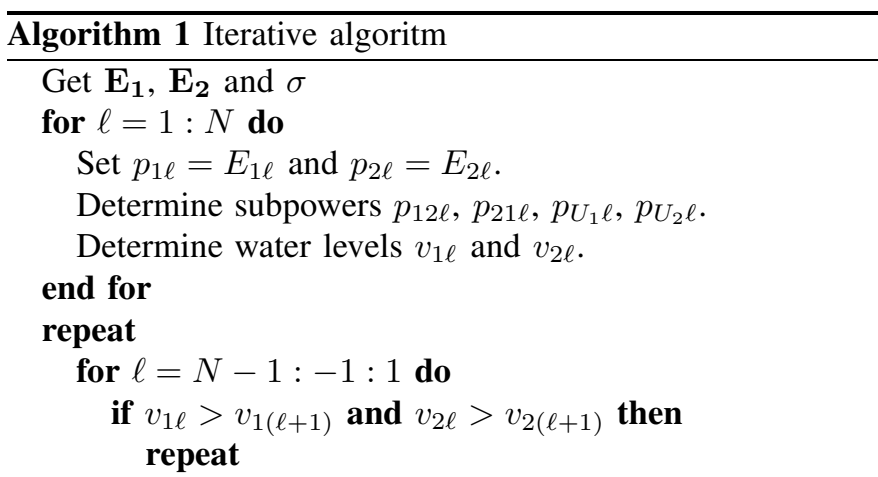

1. Equalize $v_{1 \ell}$ and $v_{1(\ell+1)}$ by changing $p_{1 \ell}$ and $p_{1(\ell+1)}$ and updating the subpowers for $\ell$ th and $(\ell+1)$ st slots.

2. Equalize $v_{2 \ell}$ and $v_{2(\ell+1)}$ by changing $p_{2 \ell}$ and $p_{2(\ell+1)}$ and updating the subpowers for $\ell$ th and $(\ell+1)$ st slots.

until $v_{1 \ell}=v_{1(\ell+1)}$ and $v_{2 \ell}=v_{2(\ell+1)}$.

else if $v_{1 \ell}>v_{1(\ell+1)}$ then

Equalize $v_{1 \ell}$ and $v_{1(\ell+1)}$ by changing $p_{1 \ell}$ and $p_{1(\ell+1)}$ and updating the subpowers for $\ell$ th and $(\ell+1)$ st slots.

else if $v_{2 \ell}>v_{2(\ell+1)}$ then

Equalize $v_{2 \ell}$ and $v_{2(\ell+1)}$ by changing $p_{2 \ell}$ and $p_{2(\ell+1)}$ and updating the subpowers for $\ell$ th and $(\ell+1)$ st slots.

end if

end for

until $v_{1}$ and $v_{2}$ are non-decreasing vectors.

lated similarly to $\mathbf{P 1}$ as

$$
\begin{aligned}
\mathbf{P 2}: \underset{\mathbf{p}}{\max } & \sum_{i=1}^{N} R_{i} \\
\text { s.t. } & \sum_{i=1}^{\ell} p_{12 i}+p_{U_{1} i} \leq \sum_{i=1}^{\ell} E_{1 i}, \\
& \sum_{i=1}^{\ell} p_{2 i} \leq \sum_{i=1}^{\ell} E_{2 i}, \\
& R_{i} \leq \frac{1}{2} \log \left(1+p_{12 i}\right), \\
& R_{i} \leq \frac{1}{2} \log \left(\frac{\tilde{S}_{i}}{\sigma^{2}}\right), \\
& p_{12 i}, p_{U_{1} i}, p_{U_{2}} \geq 0, \quad i=1, \ldots, N,
\end{aligned}
$$

where

$$
\tilde{S}_{i}=\sigma^{2}+h_{10} p_{1 i}+p_{2 i}+2 \sqrt{h_{10} p_{U_{1} i} p_{U_{2}} i} .
$$

The KKT conditions for the relay model can be obtained as

$$
\begin{aligned}
\gamma_{1 i}+\gamma_{2 i} & =1, \\
\sum_{\ell=i}^{N} \lambda_{1 \ell} & \geq \frac{\gamma_{2 i} h_{10}}{2 \tilde{S}_{i}}+\frac{\gamma_{1 i}}{2\left(1+p_{12 i}\right)},
\end{aligned}
$$




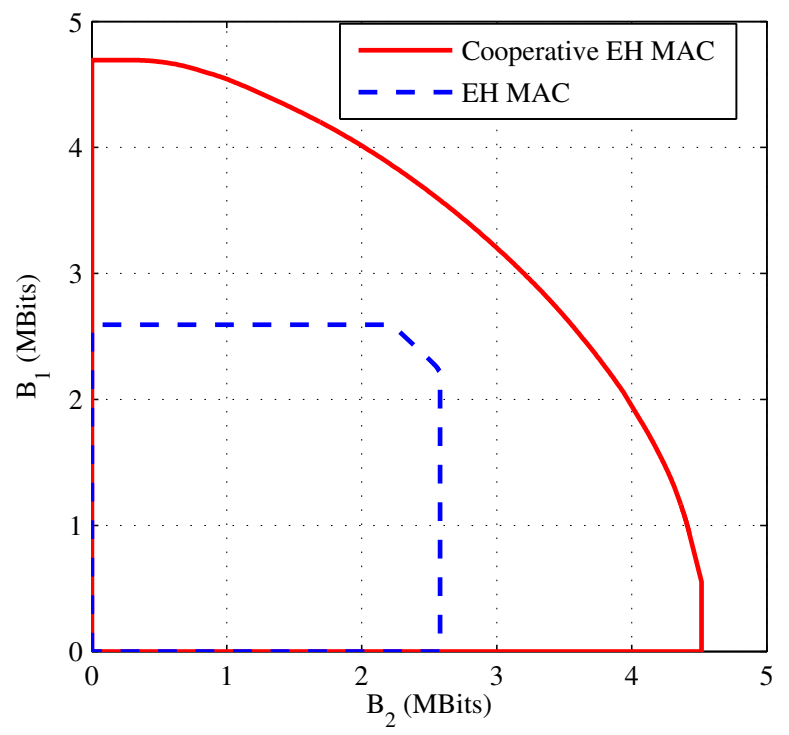

Fig. 2. Achievable rate regions of cooperative and non-cooperative strategies over the MAC with identical energy harvesting patterns.

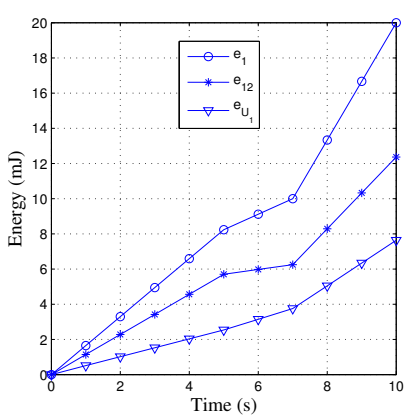

(a)

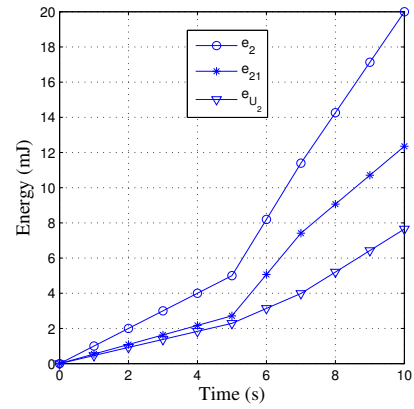

(b)
Fig. 3. Throughput maximizing transmission strategy from Fig. 2 for a) user 1 and $b$ ) user 2 .

$$
\begin{gathered}
\sum_{\ell=i}^{N} \lambda_{1 \ell} \geq \frac{\gamma_{2 i}\left(h_{10}+\sqrt{\frac{h_{10} p_{U_{2} i}}{p_{U_{1} i}}}\right)}{2 \tilde{S}_{i}}, \\
\sum_{\ell=i}^{N} \lambda_{2 \ell} \geq \frac{\gamma_{2 i}\left(1+\sqrt{\frac{h_{10 p_{U_{1} i}}}{p_{U_{2} i}}}\right)}{2 \tilde{S}_{i}} .
\end{gathered}
$$

The steps used in the solution, and the resulting algorithm are similar to those for $\mathbf{P 1}$, and are therefore omitted due to space limitations. We will present the results of our relay algorithm, and compare the achievable rate to [14], in the next section.

\section{Simulation Results}

In this section, we demonstrate that user cooperation based on energy diversity significantly improves the achievable departure region of a MAC and the throughput of a relay channel, under energy harvesting constraints. In Figs. 2 and 4, we compare the achievable departure region of the proposed cooperative energy harvesting MAC model with the capacity region

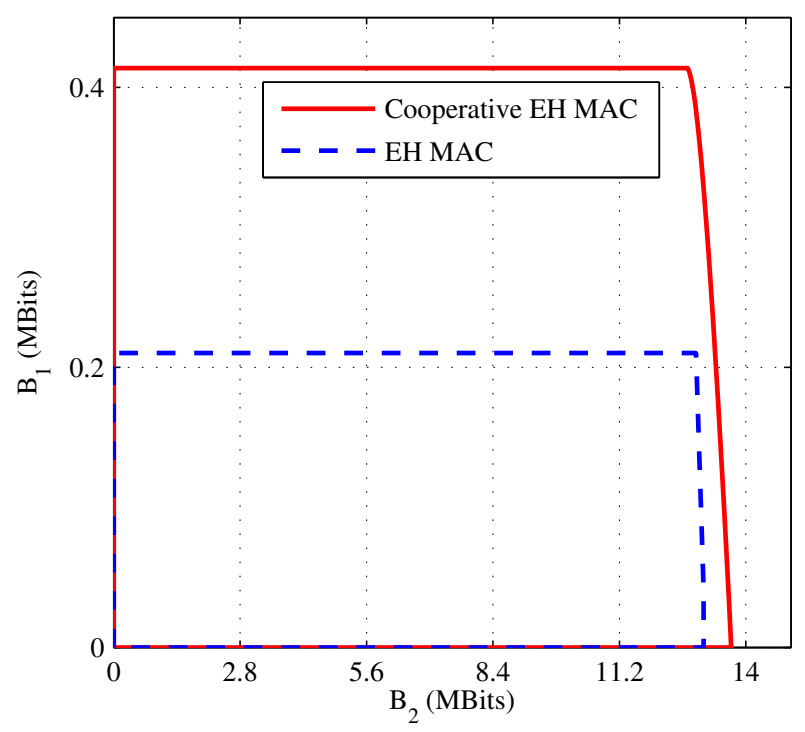

Fig. 4. Achievable rate regions of cooperative and non-cooperative strategies over the MAC with identical asymmetric energy harvesting patterns.

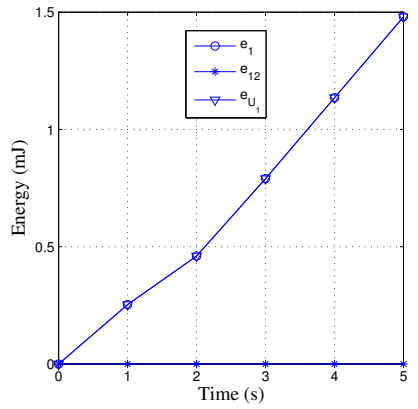

(a)

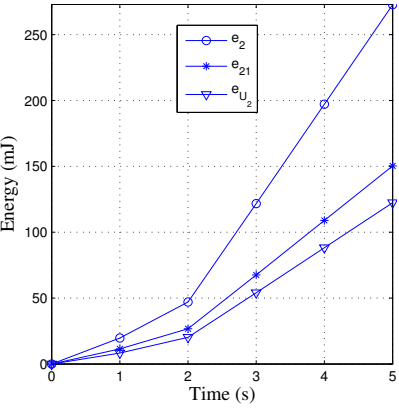

(b)
Fig. 5. Throughput maximizing transmission strategy from Fig. 4 for a) user 1 and $b$ ) user 2

of the energy harvesting MAC (EH MAC) without cooperation obtained in [7]. For direct comparison with [7], we use capacity and achievable rate formulas for bandlimited Gaussian channels, that yield the capacity and achievable rates in bits per second. We select the bandwidth and equivalent noise variance (obtained by taking into account the bandwidth, noise spectral density and path loss) as in [7]. The Gaussian noise variances on the direct links are therefore selected as $10^{-2} \mathrm{~W}$ and the transmission bandwidth is selected as $1 \mathrm{MHz}$. For the cooperative MAC only, the inter-user channels are assumed to be AWGN channels with variance $5 \times 10^{-3} \mathrm{~W}$, which translates to inter-user links having a 3-dB SNR advantage over the direct links. In Fig. 2, the departure regions of cooperative and non-cooperative MAC are compared, for $\mathbf{E}_{\mathbf{1}}=[5,0,5,0,0$, $0,0,10,0,0] \mathrm{mJ}$ and $\mathbf{E}_{\mathbf{2}}=[5,0,0,0,0,10,0,0,5,0] \mathrm{mJ}$; and a transmission deadline of 10 seconds. Using resources which are identical to those in the non-cooperative setup, our cooperative strategy significantly enlarges the achievable departure region compared to the optimum strategy without co- 


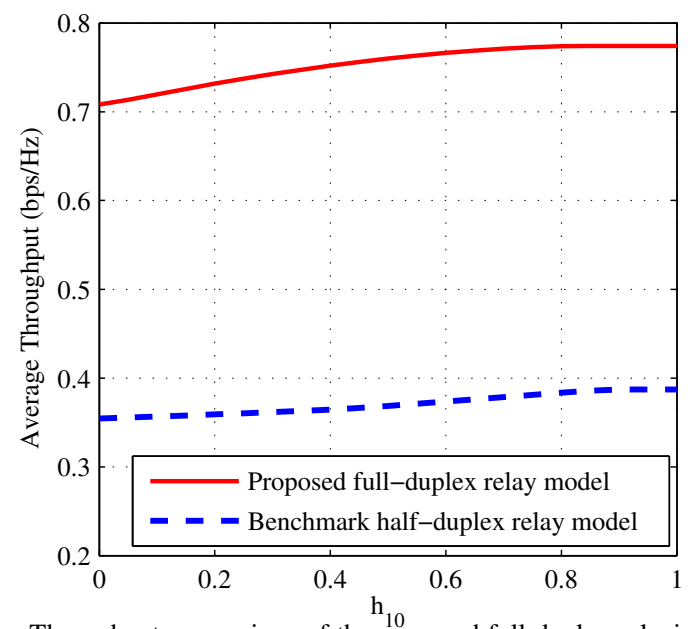

Fig. 6. Throughput comparison of the proposed full-duplex relaying strategy and the optimum strategy over the half-duplex relay channel [14].

operation. The throughput maximizing transmission strategy, corresponding to the sum departure point on the departure region boundary given in Fig. 2 is shown in Fig. 3, where $e_{m}$, with $m \in\left\{1,2,12,21, U_{1}, U_{2}\right\}$, represents the consumed energy by codeword components. Here, $p_{1}$ and $p_{2}$ (i.e., the rate of $e_{1}$ and $e_{2}$ ) do not have to be monotone increasing. However, the sum power $p_{1}+p_{2}$ is observed to be monotone increasing.

The achievable departure region of the cooperative MAC when there is significant disparity among the energy harvesting patterns, is shown in Fig. 4. The energy harvesting patterns are selected as $\mathbf{E}_{\mathbf{1}}=[0.44,0.02,0.33,0.42,0.27] \mathrm{mJ}$ and $\mathbf{E}_{\mathbf{2}}=[19.71,82.17,42.99,88.78,39.12] \mathrm{mJ}$, for a deadline of 5 seconds. We observe that, while both users do gain from cooperation, the gain is much more significant for the user at an energy disadvantage. The flat horizontal portion of the departure region corresponds to the case when user 1 only uses its energy to send its message to user 2, and user 2 relays it, in addition to sending its own data. There, the decodability condition at user 2 puts an upper bound on $B_{1}$. On the other hand, the corresponding sum-departure maximization problem transforms into maximization of $B_{2}$, as any priorities which satisfy $\mu_{2}>\mu_{1}$ result in the $B_{2}$ intercept of the departure region. The throughput (or equivalently, $B_{2}$ ) maximizing power allocation strategy is shown in Fig. 5. Unlike the findings presented in Fig. 3, when energy patterns are dissimilar in magnitude, the user with much smaller harvested energy may be forced not to transmit any of its data $\left(p_{12}=0\right)$, but to serve the other user as a cooperating partner only.

Finally, we simulate the average throughput performance of our full-duplex relaying strategy, obtained by solving $\mathbf{P 2}$. The energy harvesting pattern and channel parameters are selected to be identical to those in [14]. The results are shown in Fig. 6, along with the average throughput obtained by half-duplex relaying. We observe that, full duplex operation and coherent combining gain significantly increase the throughput of the energy harvesting relay channel.

\section{CONCLUSION}

In this paper, we solved the optimum transmit and cooperation scheduling problem in an energy harvesting cooperative MAC. We proposed a bi-directional cooperation strategy, where the common data formed by cooperation among two consecutive energy harvests is transmitted immediately, before the next harvest. We developed an iterative algorithm, which causally distributes the harvested energy in time and among cooperative codewords, thereby obtaining the optimal cooperation strategy that maximizes the departure region for any energy harvesting pattern. We demonstrated that, the diversity created by different energy arrivals at the users can be taken advantage of using cooperation, and may translate to significant rate gains, in both mutual cooperation and relaying.

\section{REFERENCES}

[1] O. Ozel and S. Ulukus, "Achieving AWGN capacity under stochastic energy harvesting," IEEE Trans. Inf. Theory, vol. 58, no. 10, pp. 64716483, Oct. 2012.

[2] O. Ozel and S. Ulukus, "AWGN channel under time-varying amplitude constraints with causal information at the transmitter," Asilomar Conference, Nov. 2011.

[3] J. Yang and S. Ulukus, "Optimal packet scheduling in an energy harvesting communication system," IEEE Trans. Commun., vol. 60, no. 1, pp. 220-230, Jan. 2012.

[4] K. Tutuncuoglu and A. Yener, "Optimum transmission policies for battery limited energy harvesting nodes," IEEE Trans. Wireless Commun., vol. 11, no. 3, pp. 1180-1189, Mar. 2012.

[5] O. Ozel, K. Tutuncuoglu, J. Yang, S. Ulukus, and A. Yener, "Transmission with energy harvesting nodes in fading wireless channels: Optimal policies," IEEE J. Sel. Areas Commun., vol. 29, no. 8, pp. 1732-1743, Sep. 2011.

[6] C. K. Ho and R. Zhang, "Optimal energy allocation for wireless communications with energy harvesting constraints," IEEE Trans. Signal Process., vol. 60, no. 9, pp. 4808-4818, Sep. 2012.

[7] J. Yang and S. Ulukus, "Optimal packet scheduling in a multiple access channel with energy harvesting transmitters," Journal of Communications and Networks, vol. 14, no. 2, pp. 140-150, Apr. 2012.

[8] Z. Wang, V. Aggarwal, and X. Wang, "Iterative dynamic water-filling for fading multiple-access channels with energy harvesting," IEEE $J$. Sel. Areas Commun., vol. 33, no. 3, pp. 382-395, Mar. 2015.

[9] J. Yang, O. Ozel, and S. Ulukus, "Broadcasting with an energy harvesting rechargeable transmitter," IEEE Trans. Wireless Commun., vol. 11, no. 2, pp. 571-583, Feb. 2012.

[10] M. A. Antepli, E. Uysal-Biyikoglu, and H. Erkal, "Optimal packet scheduling on an energy harvesting broadcast link," IEEE J. Sel. Areas Commun., vol. 29, no. 8, pp. 1721-1731, Sep. 2011.

[11] O. Ozel, J. Yang, and S. Ulukus, "Optimal broadcast scheduling for an energy harvesting rechargeable transmitter with a finite capacity battery," IEEE Trans. Wireless Commun., vol. 11, no. 6, pp. 2193-2203, Jun. 2012.

[12] K. Tutuncuoglu and A Yener, "Sum-rate optimal power policies for energy harvesting transmitters in an interference channel," Journal of Communications and Networks, vol. 14, no. 2, pp. 151-161, Apr. 2012.

[13] D. Gunduz and B. Devillers, "Two-hop communication with energy harvesting," in 4th IEEE CAMSAP, Dec. 2011.

[14] C. Huang, R. Zhang, and S. Cui, "Throughput maximization for the gaussian relay channel with energy harvesting constraints," IEEE J. Sel. Areas Commun., vol. 31, no. 8, pp. 1469-1479, Aug. 2013.

[15] Y. Luo, J. Zhang, and K. B. Letaief, "Optimal scheduling and power allocation for two-hop energy harvesting communication systems," IEEE Trans. Wireless Commun., vol. 12, no. 9, pp. 4729-4741, Sep. 2013.

[16] A. Sendonaris, E. Erkip, and B. Aazhang, "User cooperation diversity. part I. System description," IEEE Trans. Commun., vol. 51, no. 11, pp. 1927-1938, Nov. 2003.

[17] O. Kaya and S. Ulukus, "Power control for fading cooperative multiple access channels," IEEE Trans. Wireless Commun., vol. 6, no. 8, pp. 2915-2923, Aug. 2007. 